\title{
PROPIEDADES PSICOMÉTRICAS DE LA ESCALA DE EXPERIENCIAS EN RELACIONES CERCANAS -ESTRUCTURAS DE RELACIONES (ECR-RS) PARA MEDIR APEGO EN ADOLESCENTES CHILENOS
}

PSYCHOMETRIC PROPERTIES OF THE EXPERIENCES IN CLOSE RELATIONSHIPS REVISED (ECR-RS) SCALE FOR MEASURING ATTACHMENT IN CHILEAN ADOLESCENTS

DOI: 10.22199/S07187475.2015.0001.00005

KARLA TAY-KARAPAS I, MÓNICA GUZMÁN GONZÁLEZ, JAVIERA BA RRERA SILVA, DANIELA LOVYS TIRADO1, ALEXANDRA OLMOS CÓRDOVA, (UNIVERSIDAD CATÓLICA DEL NORTE, Antofagasta, Chile)

\section{RESUMEN}

Objetivo: evaluar las propiedades psicométricas de la Escala de Experiencias en Relaciones Cercanas - Estructuras de Relaciones (ECR-RS) en adolescentes chilenos entre 12 y 17 años de edad. Método: se evaluaron dos muestras independientes $(N=108 ; N=504)$ con la Escala de autoreporte $E(R-R S$, que mide el apego en sus dos dimensiones: ansiedad ante el abandono y evitación de la intimidad. Resultados: respecto de la validez de criterio, los resultados fueron distintos a lo esperado en relación con la dimensión de ansiedad ante el abandono. Si bien la dimensión de evitación de la intimidad se asoció con los constructos teóricamente propuestos en las direcciones esperadas (depresión y ansiedad), la dimensión de ansiedad no se asoció con ninguna de estas variables. Conclusiones: El ECR-RS presentó valores de confiabilidad adecuados y la estructura bidimensional propuesta originalmente fue replicada. Los hallazgos son discutidos en función de posibles explicaciones y líneas de investigación futura.

PALABRAS CLAVE: Apego, adolescencia, propiedades psicométricas ECR-RS.

\section{ABSTRACT}

Objective: To evaluate the psychometric properties of the Experiences in Close Relationships Revised (ECR-RS) Scale in Chilean adolescents between 12 and 17 years old. Method: Two independent samples ( $N=108 ; N=504$ ) were evaluated with the self-report scale ECR-RS which measures attachment in two dimensions: anxiety over abandonment and avoidance of intimacy. Results: Regarding the criterion validity, the dimension anxiety over abandonment did not show any relationship with the variables depression and anxiety, which was unusual. On the contrary, the dimension avoidance of intimacy did show a relationship with the theory as expected. Conclusions: ERC-RS showed appropriate confiability values and the proposed bidimensional structure was replicated. These findings are discussed for possible explanations and future research lines.

KEY WORDS: Attachment, adolescence, ECR-RS psychometric properties.

1. Línea de investigación en Salud Mental y contextos relacionales, Escuela de Psicología, Universidad Católica del Norte, Antofagasta - Chile. Correspondencia: Karla Tay Karapas, Escuela de Psicología Universidad Católica del Norte, Avenida Angamos \#0610, Antofagasta, Chile. E-mail: ktay@ucn.cl, Teléfono +56 (55) 2355822 
Desde su aparición, la teoría del apego de John Bowlby (1980) se ha transformado en una de las contribuciones más importantes para comprender los patrones tempranos de vinculación socio-emocional entre un individuo y sus cuidadores. Este autor, propone que el sistema de apego es fundamental para la sobrevivencia del ser humano, ya que éste busca la seguridad que su figura de cuidado le proporciona frente a condiciones de amenaza 0 inseguridad (Martínez \& Santelices, 2005). Las cualidades que adquieren dichos vínculos de apego son, por lo tanto, fuertes predictoras del desarrollo psicosocial de las personas.

En ese sentido, la medición e identificación de las características del apego se considera relevante en tanto se ha establecido que la inseguridad en el apego puede actuar como un factor de riesgo en el desarrollo de los individuos (Brando, Varela \& Zárate, 2008; DeKlyen \& Greenberg, 2008; Galán 2010; Oliva, 2004). Así, por ejemplo, se ha reportado que la inseguridad en el apego se asocia con mayores índices de depresión (Blatt, \& Levy, 2003; Cooper, Shaver, \& Collins, 1998; Kamkar, Doyle, \& Markiewicz, 2012; Kobak, Sudler, \& Gamble, 1991), menor autoestima (Gónzalez \& Méndez, 2006; Loinaz, Echeburúa \& Ullate, 2012; Wilkinson, 2004), y mayor ansiedad (Cooper et al., 1998; Lee, \& Hankin, 2009; Pacheco \& Ventura, 2009).

Tradicionalmente, han existido dos vertientes en el estudio y medición del apego (Martínez \& Santelices, 2005). La primera línea investigativa corresponde a la psicología evolutiva, iniciada por Ainsworth, Blehar, Waters \& Wall (1978), quienes elaboraron, a través de su experimento denominado la "Situación Extraña", una categorización de tres estilos de apego: seguro, evitativo y resistente-ambivalente (Garrido-Rojas, 2006). Posteriormente, Main y Solomon (como se citó en Oliva, 2004) agregaron un cuarto estilo denominado inseguro desorganizado/desorientado. La evaluación del apego en la adolescencia ha seguido esta corriente evolutiva, más cercana al psicoanálisis relacional, considerando el apego como un constructo intrapsíquico (Oliva, 2011). Desde esta perspectiva, la medición del apego se ha realizado mediante la aplicación de entrevistas y observación tanto en niños como en adolescentes y adultos.

Paralelamente, la segunda vertiente corresponde a la iniciada por la psicología social en la década del ochenta, representada por autores como Hazan y Shaver (1987) y Bartholomew y Horowitz (1991), quienes midieron cualidades del apego, patrones de conductas y sentimientos en relaciones de pareja, a través de la aplicación de cuestionarios de autorreporte (Martínez \& Santelices, 2005). Con posterioridad, estos autores conceptualizaron los estilos de apego de Ainsworth como regiones en un espacio bidimensional: la ansiedad ante el abandono y la evitación de la intimidad.

En la literatura internacional se encuentran múltiples instrumentos para medir el apego. Desde la aproximación categorial evolutiva, uno de los más utilizados en población adulta es la Adult Attachment Interview (AAI, de George, Kaplan, \& Main, 1985), la que evalúa los recuerdos del sujeto respecto de las experiencias de apego en la infancia y la valoración de éstas a través de una entrevista semi-estructurada. Para adolescentes se utiliza la Friends and Family Interview (FFI, de Steele, \& Steele, 2005) que aborda ocho dimensiones asociadas al apego en una entrevista semiestructurada. Finalmente, para la población infantil se dispone de la Child Attachment Interview (CAI, de Shmueli-Goetz, 2001), instrumento que examina la percepción que poseen los infantes sobre su cuidador, sobre sí mismo y sobre el vínculo de apego.

Desde la aproximación dimensional, más cercana a la corriente de la psicología 
social, se reportan instrumentos como el Experiences in Close Relationships (ECR, de Brennan, Clark, \& Shaver, 1998) que evalúa el apego romántico en población adulta y sitúa al sujeto en los continuos de ansiedad y de evitación. A partir de este mismo instrumento, surge la versión revisada, construida con base en la teoría de la respuesta al ítem (ECR-Rraley, Waller \& Brennan, 2000). Posteriormente, se desarrolla el ECR-RS (Fraley, Heffernan, Vicary, Brumbaugh, 2011), que mide el apego en dominios relacionales específicos (hacia la figura paterna y materna, de pareja romántica y mejor amigo).

En el marco específico de la medición del apego en niños/as y adolescentes, se cuenta con la escala Experiences in Close Relationships Scale-Revised for use with Children and Adolescents (ECR-RC, de Brenning, Soenens, Braet, \& Bosmans, 2011) para medir el apego desde una perspectiva dimensional.

En Chile, para estudiar la población infantojuvenil sólo se cuenta con la validación realizada por Felipe Lecannelier (2002) de la Child Attachment Interview (CAI, de Shmueli-Goetz, 2001). Tal antecedente, evidencia la escasez de instrumentos validados en nuestro país que permitan medir el apego en esta etapa evolutiva, y el que existe se basa en una aproximación categorial que tiene como desventaja la pérdida de información clínicamente valiosa, asimismo su aplicación requiere de un mayor tiempo destinado al entrenamiento de quien lo aplica, al proceso de recolección y análisis de datos, haciendo compleja su aplicación masiva.

Contar con un instrumento que permita medir el apego en adolescentes desde una aproximación dimensional, representa un aporte en diversos aspectos. Primero, permite contar con un instrumento, adaptado a nuestro país, que posibilite el desarrollo de investigaciones en esta etapa evolutiva. Segundo, la detección de las características del apego favorece el diseño de programas de promoción, prevención e intervención en salud mental con población adolescente. Tercero, permite disponer de un instrumento que tiene como ventajas su brevedad y la posibilidad de ser administrado de forma masiva en esta población, facilitando la obtención de datos. Por último, contribuye a la realización de estudios comparativos con otros países, lo que permitiría determinar diferencias contextuales y/o culturales en relación al apego de los adolescentes.

Con base en los antecedentes expuestos, el objetivo general del presente estudio fue evaluar las propiedades psicométricas del instrumento Experiencias en Relaciones Cercanas - Estructuras de Relaciones (ECR-RS, de Fraley et al., 2011) en adolescentes chilenos entre 12 y 17 años de edad.

\section{Teoría del Apego}

A fines de la década de los sesenta, el psicoanalista británico John Bowlby realizó un aporte significativo a la psicología evolutiva al elaborar la Teoría del Apego. Para Bowlby (1986) la vinculación afectiva es el resultado del comportamiento social de cada individuo de una especie. Es decir, el tipo de vínculo que se forma con otros individuos estará determinado por la experiencia social que se forje entre los mismos. Siguiendo esta línea, el mantenimiento firme de un vínculo es experimentado como fuente de seguridad (Bowlby, 1986). Es por esto que durante los primeros años de vida, la tarea principal es la creación de un apego seguro, de comunicación afectiva entre el bebé y el cuidador primario (Schore, 2009), capaz de entregar una sensación de seguridad, protección, disponibilidad y responsividad ante demandas de orden biológico y psicológico (Oliva, 2004). 
Para Bowlby (1986), el funcionamiento sano de la personalidad en cada edad refleja, por un lado, la capacidad de un individuo para reconocer figuras adecuadas capaces de proporcionarle una base segura $y$, por otro lado, su capacidad para colaborar con tales figuras en relaciones mutuamente gratificantes.

Como sabemos, la adolescencia es un período de profundas transformaciones cognitivas, emocionales y sociales que tendrán una repercusión directa sobre el significado y expresión de los procesos de apego (Oliva, 2011). A lo largo de esta etapa, el adolescente se enfrenta a nuevos desafíos personales y para ello requerirá del apoyo de figuras significativas para superar esta crisis, que supone la redefinición de las relaciones de dependencia de las figuras de apego. Esto, se condice con lo señalado por Cassidy (2008), quien indica que la adolescencia es una etapa de transición de las dependencias de las relaciones parentales a las de los pares. El modo en que el adolescente conforme dichas relaciones con sus pares, estaría en estrecha relación con el estilo de apego que haya construido en su primera infancia (Oliva, 2011), considerando que aquellos adolescentes que formaron vínculos seguros en sus familias tienden a mantener este mismo tipo de apego en otras relaciones (Delgado, Oliva \& SánchezQueija, 2011). Sin embargo, es importante señalar que diversos factores intervienen al momento de establecer el proceso de vinculación con una figura de apego, ya que, como señala Bowlby (1986), los patrones de apego dependen parcialmente de la edad, el sexo, las circunstancias presentes, así como de las experiencias de apego anteriores.

Bowlby (1980) plantea que la teoría del apego permite explicar diversas alteraciones emocionales y de la personalidad. Se ha establecido que la inseguridad del apego se asocia a la presencia de sintomatología depresiva (Fraley, et al., 2011; González \&
Méndez, 2006; Pardo, Sandoval \& Umbarila, 2004). En la misma línea, Cooper et al., (1998) y Kamkar et al., (2012), indican que adolescentes con apego seguro poseen niveles más bajos de depresión, adolescentes evitativos obtienen un nivel intermedio y adolescentes ansiosos/ambivalentes puntúan los niveles más altos del grupo. Asimismo, la inseguridad del apego ha sido asociada también a la presencia de sintomatología ansiosa (Cooper et al., 1998; Lee, \& Hankin, 2009; Pacheco \& Ventura, 2009).

\section{Medición del Apego en la Adolescencia}

De manera paralela al estudio de los aspectos teóricos del apego, parte de los investigadores ha abordado la medición de éste como eje fundamental para aportar al desarrollo de la teoría, tanto conceptual como empíricamente. Algunos de ellos son: Ainsworth et al. (1978), Brennan et al., (1998), Target, Fonagy \& Shumueli-Goetz (2003), entre otros.

Considerando que el apego es un sistema que se manifiesta a lo largo de la vida, se han creado instrumentos de medición que permiten obtener resultados en cada una de las etapas evolutivas del ser humano, adaptados según las características propias de la niñez, la adolescencia y la adultez. Respecto de la adolescencia, existen diversas maneras de medir el apego, sin embargo, los instrumentos son escasos. Román (2011), explica que el modo de evaluar el apego se relaciona directamente con la edad del sujeto, ya que durante los primeros años es posible observar de manera directa la activación del sistema de apego, mientras que con el transcurso del tiempo, éste se desactiva y las personas crean representaciones mentales acerca de sus vínculos tempranos.

En Chile, existe escasez de estudios sobre apego en la adolescencia, sin embargo, se tiene evidencia de la aplicación 
de instrumentos como el Relationship Questionnaire (Bartholomew \& Horowitz, 1991) y las subescalas del Adolescent Attachment Questionnaire (West, Spreng, Sheldon-Keller \& Adam, 1998), ambos utilizados por González y Méndez (2006) en una investigación orientada a evaluar la relación entre depresión, autoestima y apego. Asimismo, Lecannelier (2002) presenta la versión del Child Attachment Interview (CAl, de Shmueli-Goetz, 2001) para niños entre 7 y 13 años. En la misma línea, Escobar y Santelices, (2013) aplicaron la Friends and Family Interview (FFI, de Steele \& Steele, 2005) a la población adolescente. Ambos instrumentos pertenecen a una aproximación categorial.

La adaptación de instrumentos que pertenezcan a la aproximación dimensional del apego y que posea una modalidad de auto-reporte ha sido más bien escasa, contando sólo con la validación del ECR (Spencer, Guzmán, Fresno \& Ramos, 2013), pero que está diseñado para población adulta.

Para este estudio, se ha escogido la escala Experiencias en Relaciones Cercanas- Estructuras de Relaciones (ECRRS, de Fraley et al., 2011) como instrumento a validar dadas sus buenas propiedades psicométricas, su brevedad y porque permite medir el apego en diversos dominios relacionales (en el caso del presente estudio apego hacia la madre y el padre). Es importante mencionar que la escala Experiences in Close RelationshipsRelationship Structures (ECR-RS de Fraley et al., 2011), fue creada con el fin de mejorar las limitaciones psicométricas y teóricas de los instrumentos para medir apego, tomando como base antecedentes teóricos que indican que los modelos internos de apego (MOl) pueden variar dependiendo de la figura con la que se establece una relación cercana y la experiencia que deriva de ésta.
En síntesis y considerando los antecedentes expuestos, el objetivo general del estudio fue evaluar las propiedades psicométricas de la Escala de Experiencias en Relaciones Cercanas - Estructuras de Relaciones (de Fraley et al., 2011), en adolescentes chilenos entre 12-17 años de edad, a fin de medir las características del apego hacia las figura materna y paterna. Para ello, se definieron los siguientes objetivos específicos:

1. Analizar la estructura dimensional del ECR-RS para la evaluación del apego hacia los padres en adolescentes entre 12 y 17 años.

2. Evaluar la consistencia interna.

3. Evaluar la validez de criterio, estableciendo relación con síntomas de depresión y ansiedad estado y rasgo.

\section{Hipótesis}

1. Se espera que el ECR-RS posea la estructura bidimensional propuesta, es decir, conformada por los factores de ansiedad ante el abandono y evitación de la intimidad.

2. Se espera que exista asociación entre las dimensiones del apego y la presencia de síntomas depresivos. Específicamente, se hipotetiza que a mayor ansiedad y/o evitación del apego, habrá mayores índices de sintomatología depresiva.

3. Se espera que exista asociación entre las dimensiones de apego y ansiedad. Específicamente, se hipotetiza que a mayor ansiedad y/o evitación del apego, habrá mayores índices de ansiedad. 
MÉTODO

Participantes

Se evaluaron dos muestras no probabilísticas independientes de adolescentes, siendo además la muestra 2 estratificada por sexo y tipo de establecimiento educacional (municipalizados, particulares subvencionados y particulares pagados) de la ciudad de Antofagasta, Chile. Los criterios de inclusión fueron tener entre 12 y 17 años de edad, ser chileno/a, contar con la autorización y firma del consentimiento informado por parte de sus padres y/o apoderados, y posterior asentimiento de cada participante. Quedaron excluidos del estudio aquellos colegios cuyos objetivos están orientados al trabajo con alumnos que poseen necesidades educativas especiales.

Para conformar la muestra, se seleccionaron cuotas de la cantidad de estudiantes pertenecientes a los tres tipos de establecimientos educacionales, a fin de representar la proporción efectiva de cada uno de ellos.

Muestra 1. Se evaluó un total de 108 adolescentes de la ciudad de Antofagasta, de los cuales, el $49.1 \%$ fueron hombres y el $50.9 \%$ mujeres. El promedio de edad fue 14.9 años $(D E=1.76)$. Respecto de la distribución según tipo de establecimiento, el $54.6 \%$ de los jóvenes asisten a establecimientos municipalizados, el $20 \%$ a establecimientos particulares subvencionados y el $25 \%$ a establecimientos particulares pagados.

Muestra 2. Compuesta por 510 adolescentes, distribuidos en 257 mujeres $(50.4 \%)$ y 253 hombres $(49.6 \%)$, con un promedio de edad de 14.6 años ( $D E=$ 1.67). En cuanto a la distribución por tipo de establecimiento, el $42 \%$ pertenece a municipales, el $40 \%$ a particulares subvencionados y el $17.8 \%$ a particulares pagados.

\section{Instrumentos}

Además de la caracterización sociodemográfica (edad, sexo y nivel socioeconómico) se utilizaron los siguientes instrumentos de autorreporte:

Experiences in Close Relationships Relationship Structures (ERC-RS). Diseñada, por Fraley et al., (2011), es una escala compuesta nueve ítems distribuidos en dos sub-escalas, de evitación y ansiedad, donde los ítems 1, 2, 3, 4, 5 y 6 evalúan la dimensión evitación de la intimidad y los ítems 7, 8 y 9 la dimensión ansiedad ante el abandono. Los mismos nueve ítems se utilizan para medir apego en cuatro dominios relacionales: hacia la figura materna y paterna, la pareja romántica y el mejor amigo/a, obteniendo puntuaciones independientes para cada uno de los dominios en las dos dimensiones descritas. Las respuestas oscilan entre 1 y 7 en formato Likert. (1= "Totalmente en desacuerdo" hasta $7=$ "Totalmente de acuerdo"). En este instrumento, puntajes más altos se interpretan como niveles más altos de ansiedad y/o evitación y por ende, mayor inseguridad del apego.

Para efectos de este estudio se ocuparon las dos escalas que evalúan las dimensiones del apego hacia la figura materna y paterna, es decir, se consideró un total de 18 ítems.

Escala de Depresión del Centro para Estudios Epidemiológicos (CES-D). Validada en Chile para población adolescente y adulta (Gempp, Avendaño \& Muñoz, 2004), mide los principales síntomas del trastorno depresivo a través de 20 ítems. Éstos se presentan en formato Likert de 4 frecuencias ( $0=$ "Rara vez o una vez" [1 día o menos], hasta 3= "La mayor parte del tiempo" [5 a 7 días]) tomando como referente la vida del sujeto durante la última semana. En cuanto a sus propiedades psicométricas, se reporta una confiabilidad de .87 mediante el alfa de Cronbach. Este instrumento posee un puntaje de corte de 
24 en su validación chilena (Gempp et al., 2004), indicando que a medida que aumenta este valor, mayor es el nivel actual de depresión.

Cuestionario de Ansiedad Estado Rasgo. Validado en Chile para población adolescente y adulta (Vera-Villarroel, CelisAtenas, Córdova-Rubio, Buela-Casal \& Spielberger, 2007). Está orientado a la medición de la ansiedad desde dos escalas independientes, la primera relacionada con la ansiedad como condición transitoria denominada Ansiedad-Estado; la segunda mide la ansiedad como un concepto estable denominada Ansiedad-Rasgo. instrumento está compuesto por 40 ítems, 20 para cada subescala y la modalidad de respuesta es de formato Likert de cuatro categorías para la escala de Ansiedad Estado (desde $0=$ "Nada" a $3=$ "Mucho") y cuatro categorías para las escala de Ansiedad Rasgo (desde 0= "Casi Nunca" a $3=$ "Casi siempre"). A medida que aumenta el puntaje obtenido mayor es el nivel de ansiedad para ambas subescalas. La versión chilena cuenta con una confiabilidad de .92 para la escala de Ansiedad-Estado y .87 para la escala de Ansiedad-Rasgo.

\section{Procedimiento}

El proceso de validación constó de cinco etapas. La primera, consistió en la traducción del instrumento, mediante un diseño de traducción cruzada. El instrumento fue traducido al castellano por una persona bilingüe, luego se realizó la traducción inversa, es decir, nuevamente al inglés por tres personas bilingües con formación en psicología, quienes discutieron la mejor traducción con la finalidad de comprobar la concordancia de los enunciados de cada ítem.

En una segunda etapa, un grupo de jueces expertos compuesto por cuatro psicólogos especializados en el trabajo con adolescentes evaluaron cada ítem de manera independiente, de acuerdo a su redacción, significado y la relación del contenido del test con el constructo que se midió. Además, discutieron en relación con la edad pertinente para la aplicación del instrumento. A partir de esta evaluación, emergieron sugerencias que fueron incorporadas en la versión final del ECRRS.

En un tercer momento, se realizó la entrevista cognitiva a 15 adolescentes que cumpliesen los mismos criterios de inclusión definidos previamente con la finalidad de examinar en qué medida la escala mide lo que dice medir, a través del análisis del proceso mental de responder los reactivos (Smith-Castro \& Molina, 2011). Ésta, se realizó en un ambiente controlado, previo consentimiento de los padres y asentimiento de los jóvenes.

En una cuarta etapa, se aplicó la prueba piloto a un grupo conformado por 108 adolescentes cuyas características concordaban con los criterios de inclusión estipulados para la investigación. Esto con la finalidad de evaluar el comportamiento del instrumento y realizar eventuales ajustes a éste.

Finalmente, en una quinta etapa, se aplicó el instrumento en su versión final a la muestra dos. Para ello, se realizó el contacto con el equipo directivo de los distintos establecimientos educacionales obteniendo la autorización para iniciar el proceso. Posteriormente, se asistió a una reunión con los padres y apoderados de los adolescentes, donde se dio a conocer el objetivo de la investigación y se les solicitó completar el consentimiento informado y la encuesta sociodemográfica. A continuación, se acudió al establecimiento a fin de acceder a los estudiantes, informándoles de los objetivos del estudio, procediendo con la lectura de la carta de asentimiento, enfatizando el carácter voluntario y confidencial de la participación. A quienes asintieron en participar se les entregó el set de instrumentos en papel. 


\section{Estrategia de Análisis de Datos}

El análisis de datos fue realizado con el programa SPSS en su versión número $20 \mathrm{y}$ el programa EQS 5.0 (Bentler, 2005). Se hizo un examen preliminar de los datos, a fin de detectar y manejar casos ausentes y atípicos, excluyéndose cuatro casos en el proceso. Además, y a fin de maximizar la potencia estadística de los análisis, se reemplazaron los valores perdidos con el método de Esperanza-Maximización (EM), resguardando que los ítems no superasen más del $5 \%$ de valores perdidos y que fuesen datos faltantes al azar (Harel, Zimmerman, \& Dekhtyar, 2008; Tabachnick \& Fidell, 2007). Posteriormente, se realizó el análisis descriptivo de los datos y se evaluaron las diferencias existentes en las dimensiones del apego de acuerdo al sexo, mediante la prueba $t$ de Student.

Para el examen de la estructura factorial del instrumento, preliminarmente se realizó un análisis factorial exploratorio (AFE) con el método de extracción de ejes principales y rotación oblicua en la muestra uno (pilotaje). Con la muestra dos, se realizó un análisis factorial confirmatorio (AFC).

Luego de esto se evaluó la consistencia interna del instrumento, mediante el alfa de
Cronbach. Finalmente, para la evaluación de la validez de criterio, se realizó un análisis de correlación entre las dimensiones del ECR-RS y las variables consideradas (sintomatología depresiva, ansiedad rasgo y ansiedad estado) en la segunda muestra.

\section{RESULTADOS}

\section{Muestra 1 \\ Análisis factorial exploratorio}

El análisis factorial exploratorio (AFE) se llevó a cabo utilizando el método de extracción de factorización de ejes principales y con rotación Varimax. De manera previa se utilizó el test de esfericidad de Bartlett $(p=0.000)$ y el índice $\mathrm{KMO}=.66$, para la figura materna y $\mathrm{KMO}=$ .77 para la figura paterna, con el fin de evaluar la pertinencia de realizar este análisis. Para determinar el número de factores a extraer, se consideraron como criterios los autovalores mayores que 1 y el gráfico de sedimentación. Según estos aspectos, serían dos los factores que darían cuenta de la estructura de datos, explicando un $54.8 \%$ de la varianza. Los ítems incluidos fueron los 9 para cada dominio relacional de ambas subescalas obteniéndose un $44.9 \%$ para figura materna y $56 \%$ figura paterna.

TABLA 1.

Factores del ECR-RS Relacionados a los Dominios de Figura Materna y Paterna.

\begin{tabular}{|c|c|c|c|c|}
\hline \multirow[b]{2}{*}{ Ítems } & \multicolumn{2}{|c|}{ Figura Materna } & \multicolumn{2}{|c|}{ Figura Paterna } \\
\hline & Evitación & Ansiedad & Evitación & Ansiedad \\
\hline 1. Me ayuda mucho recurrir a esta persona en momentos difíciles. & .67 & & .85 & \\
\hline 2. Frecuentemente converso sobre mis problemas y preocupaciones con esta persona. & .91 & & .91 & \\
\hline 3. Hablo de mis cosas personales con esta persona. & .72 & & .82 & \\
\hline 4. Se me hace fácil estar cerca emocionalmente de esta persona. & .35 & & .69 & \\
\hline 5. Me siento incómodo/a contando las cosas que a mí me pasan a esta persona. & & .31 & & .39 \\
\hline 6. Prefiero no contarle a esta persona cómo me siento realmente. & & .28 & & .49 \\
\hline 7. Frecuentemente me preocupa no importarle realmente a esta persona. & & .66 & & .64 \\
\hline 8. Me asusta que esta persona pudiera abandonarme. & & .76 & & .78 \\
\hline 9. Me preocupa no importarle a esta persona tanto como ella me importa a mí. & & .91 & & .88 \\
\hline
\end{tabular}

Tal como se observa en la Tabla 1 , se identifica un factor que agrupa los ítems 1 , 2, 3 y 4 que corresponden a la subescala de evitación $y$, un segundo factor, que incluye los ítems 7,8 y 9, que conforman la subescala de ansiedad. Los ítems 5 y 6 , que pertenecen a la escala de evitación, cargan en la escala de ansiedad en los dominios de figura materna y paterna. 
Tal como se observa en la Tabla 1 , se identifica un factor que agrupa los ítems 1 , 2, 3 y 4 que corresponden a la subescala de evitación y, un segundo factor, que incluye los ítems 7, 8 y 9, que conforman la subescala de ansiedad. Los ítems 5 y 6 , que pertenecen a la escala de evitación, cargan en la escala de ansiedad en los dominios de figura materna y paterna.

\section{Consistencia interna del ECR-RS}

Se evaluó mediante el estadístico alfa de Cronbach para cada sub-escala respecto de los dos dominios relacionales y para la escala general, tal como se observa en la Tabla 2. El índice de consistencia interna para la subescala de evitación fue de .76 y de .90 para la de ansiedad.

TABLA 2.

Valores de alfa de Cronbach para cada Dominio Relacional para el ECR-RS.

\begin{tabular}{lccc}
\hline Sub-escalas & $\begin{array}{c}\text { Figura } \\
\text { Materna }\end{array}$ & $\begin{array}{c}\text { Figura } \\
\text { Paterna }\end{array}$ & Total \\
\hline Ansiedad & .82 & .83 & .90 \\
Evitación & .61 & .67 & .76 \\
\hline$(\mathrm{n}=108)$ & & &
\end{tabular}

Diferencias en las dimensiones del apego según sexo

Se ocupó la prueba $t$ de Student para muestras independientes a fin de evaluar diferencias en las dimensiones del apego en función del sexo. Tal como se aprecia en la Tabla 3, no se detectaron diferencias estadísticamente significativas entre hombres y mujeres.

TABLA 3.

Resultados de la prueba $t$ para Hombres y Mujeres.

\begin{tabular}{lccc}
\hline Variable & $\begin{array}{c}M(D E) \\
\text { Hombres }\end{array}$ & $\begin{array}{c}M(D E) \\
\text { Mujeres }\end{array}$ & $t$ \\
\hline $\begin{array}{c}\text { Ansiedad } \\
\text { Figura Materna }\end{array}$ & $3.88(2.11)$ & $3.80(2.12)$ & .18 \\
Figura Paterna & $3.58(2.07)$ & $3.96(2.14)$ & -.90 \\
$\quad$ Evitación & & & \\
Figura Materna & $3.10(0.88)$ & $3.19(1.10)$ & -.45 \\
Figura Paterna & $3.76(1.29)$ & $4.09(1.24)$ & -1.35 \\
\hline${ }^{*} p<.05$ & & &
\end{tabular}

Muestra 2

Estructura factorial

A fin de evaluar la validez de constructo del ECR-RS, se realizó un Análisis Factorial Confirmatorio (AFC) para examinar el ajuste de los datos al modelo de dos dimensiones: ansiedad ante el abandono y evitación de la intimidad. Para ello se incluyeron inicialmente los 9 ítems de cada dominio relacional por separado (figura materna y figura paterna). Además, al especificar los modelos, se fijó en 1 la carga de uno de los ítems en cada factor.

El ajuste del modelo inicial (con los 9 ítems) evidenció un bajo ajuste a los datos, tanto en relación con la figura materna, $(X 2(27, N=510)=190.11, p<.001 ; X 2 / g \mid$ $=7.04 ; \mathrm{NFI}=.88 ; \mathrm{NNFI}=.86 ; \mathrm{CFI}=.90$; RMSEA $=.109,90 \% \mathrm{Cl}[.094, .124]$, como en relación con la figura paterna, $(X 2(27, N$ $=510)=197.10, p<.001 ; X 2 / g \mid=7.3 ; \mathrm{NFI}$ $=.89$; NNFI $=.87$; CFI $=.91$; RMSEA $=.111$, $90 \% \mathrm{Cl}[.097, .126]$.

Las cargas factoriales fueron todas significativas $(p<.01)$. Sin embargo, al examinar los índices de modificación propuestos por el programa, en ambos dominios relacionales se observó que los ítems 5 y 6 tenían saturaciones en los dos factores: evitación (dimensión a la que originalmente pertenecían) y ansiedad.

Considerando estos antecedentes, se decidió re-estimar un segundo modelo, excluyendo los reactivos 5 y 6 . El ajuste de este nuevo modelo (con 7 ítems) evidenció un mejor ajuste a los datos, tanto en relación con la figura materna, $(X 2(14, N$ $=510)=49.76, p<.001 ; x 2 / g \mid=3.44 ; N F I$ $=.96$; NNFI =.96; CFI =.97; RMSEA =.071, $90 \% \mathrm{Cl}[.050, .093]$, como en relación con la figura paterna, $(X 2(14, N=510)=70.49, p<$ $.001 ; \mathrm{X} / \mathrm{gl}=5.04 ; \mathrm{NFI}=.96 ; \mathrm{NNFI}=.95 ; \mathrm{CFI}$ $=.97 ; \mathrm{RMSEA}=.089,90 \% \mathrm{Cl}[.069, .110]$. 
Las cargas factoriales de este segundo modelo fueron adecuadas tanto en términos de significación como de magnitud con saturaciones entre .59 y .93 para el dominio de la figura materna y entre .63 y .93 para la figura paterna, valores los que superan el mínimo de .40 considerado aceptable (Kline, 2005).

\section{Análisis de consistencia interna}

Como se presenta en la Tabla 4, se evaluó la consistencia interna de la escala, a partir del estadístico alfa de Cronbach. Los valores para la muestra 2 son .90 para la dimensión de ansiedad y de .83 para la dimensión de evitación, aumentando a .84 al eliminar los ítems 5 y 6 .

TABLA 4.

Valores de alfa de Cronbach para cada Dominio Relacional.

\begin{tabular}{cccc}
\hline Sub-escalas & Figura Materna & Figura Paterna & Total \\
\hline Ansiedad & .80 & .85 & .90 \\
Evitación & .80 & .78 & .83 \\
Evitación (sin ítem 5 y 6) & .84 & .86 & .84 \\
\hline (N=510) & & &
\end{tabular}

Diferencias en las dimensiones del apego según sexo

Con el fin de evaluar diferencias en las dimensiones del apego en función del sexo se utilizó la prueba $t$ de Student para muestras independientes. Tal como se observa en la Tabla 5, se detectaron diferencias en la dimensión de evitación para ambas figuras, siendo los hombres quienes presentan mayor evitación hacia la figura materna, mientras que las mujeres presentan mayor evitación hacia la figura paterna.

TABLA 5.

Resultados de la Prueba t para Hombres y Mujeres.

\begin{tabular}{lccc}
\hline \multicolumn{1}{c}{ Variable } & $M(D E)$ Hombres & $M(D E)$ Mujeres & $t$ \\
\hline Ansiedad & & & \\
Figura Materna & $3.84(2.05)$ & $3.95(2.21)$ & -.55 \\
Figura Paterna & $3.82(2.10)$ & $4.02(2.23)$ & -1.06 \\
Evitación & & & \\
Figura Materna & $3.37(1.35)$ & $3.02(1.35)$ & $2.91^{*}$ \\
Figura Materna (Sin ítem 5 y 6) & $3.21(1.69)$ & $2.73(1.43)$ & $3.61^{*}$ \\
Figura Paterna & $3.70(1.38)$ & $4.08(1.38)$ & $-3.05^{*}$ \\
Figura Paterna (Sin ítem 5 y 6) & $3.68(1.58)$ & $4.12(1.65)$ & $-2.98^{*}$ \\
\hline${ }^{*}$ p.05 & & &
\end{tabular}

\section{Validez de criterio}

Se realizó el Análisis de Correlación Bivariado entre las dimensiones del ECR$\mathrm{RS}$, para ambos dominios relacionales, con las variables de depresión, ansiedad estado y ansiedad rasgo. Tal como se observa en la Tabla 6, hubo asociación significativa en las direcciones esperadas, entre la dimensión de evitación (figura materna y paterna) y todas las variables mencionadas $(p<.05)$. Sin embargo, no ocurrió lo mismo con la dimensión de ansiedad, que no se correlacionó de manera significativa con ninguna de las variables. 
TABLA 6.

Correlaciones entre las Dimensiones del Apego según Figura y las Variables Depresión, Autoestima y Ansiedad.

\begin{tabular}{lcccccc}
\hline \multicolumn{1}{c}{ Variables } & $\begin{array}{c}\text { Evitación } \\
\text { Figura } \\
\text { Materna }\end{array}$ & (Sin ítem 5 y 6) & $\begin{array}{c}\text { Evitación } \\
\text { Figura } \\
\text { Paterna }\end{array}$ & (Sin ítem 5 y 6) & $\begin{array}{c}\text { Ansiedad } \\
\text { Figura } \\
\text { Materna }\end{array}$ & $\begin{array}{c}\text { Ansiedad } \\
\text { Figura } \\
\text { Paterna }\end{array}$ \\
\hline Depresión & $.25^{* *}$ & $.19^{* *}$ & $.25^{* *}$ & $.22^{* *}$ & .07 & .07 \\
Ansiedad Rasgo & $.25^{* *}$ & $.17^{* *}$ & $.23^{* *}$ & $.24^{* *}$ & .01 & .00 \\
Ansiedad Estado & $.24^{* *}$ & $.22^{* *}$ & $.27^{* *}$ & $.22^{* *}$ & .07 & .07 \\
\hline${ }^{* *}$ La correlación es significativa al nivel 0.01 & & & & & \\
${ }^{*}$ La correlación es significante al nivel 0.05 & & & &
\end{tabular}

\section{DISCUSION}

El objetivo de la investigación fue evaluar las propiedades psicométricas de la Escala Experiencias en Relaciones Cercanas Estructuras de Relaciones (ECR-RS) (Fraley et al., 2011) en adolescentes chilenos entre 12 a 17 años de edad para evaluar el apego hacia la figura materna y paterna.

En el análisis factorial, los ítems se agruparon en las dos dimensiones esperadas: ansiedad y evitación, por ende, se acepta la primera hipótesis del estudio. Sin embargo, se eliminaron dos ítems (reactivos 5 y 6 ), dado que a pesar de ser analizados y redactados nuevamente para la aplicación de la muestra 2, continuaron cargando en ambas dimensiones. Además, el análisis de fiabilidad apoyó el carácter prescindible de estos ítems, dado que el valor de consistencia interna de la escala aumentó al eliminarlos, tanto en el instrumento total como en ambas subescalas.

Por otra parte, en relación a la evaluación de la consistencia interna, los valores obtenidos fueron apropiados, siendo la escala de ansiedad la que obtuvo índices más altos.

En cuanto a las diferencias por sexo, en las dimensiones de ansiedad y evitación de nuestro estudio, los hombres reportaron mayores niveles de evitación hacia la figura materna, mientras que las mujeres reportaron mayores niveles de evitación hacia la figura paterna. Lo anterior, pudiese explicarse a partir de los procesos y tareas evolutivas propias de la adolescencia que influirían en los vínculos de apego. Así, es posible suponer que se tenderá a priorizar relaciones de apego con aquella figura con la cual el adolescente comparte ciertas características y por ende, se identifique. Así por ejemplo, los hombres tenderán a identificarse con su figura paterna considerando el rol de género que comparten.

En cuanto a la dimensión de ansiedad, no se encontraron diferencias significativas entre hombres y mujeres. Los datos son diferentes de acuerdo a lo planteado teóricamente, ya que se espera que las mujeres manifiesten mayores signos de ansiedad a diferencia de los hombres de quienes se espera mayores signos de evitación (Donbaeka \& Elklitb, 2013).

En cuanto a la validez de criterio, referidas a las hipótesis 2 y 3 , estas se sostienen solo parcialmente, dado que existen diferencias en el patrón de resultados para las dimensiones de ansiedad y evitación. De acuerdo a lo esperado, la dimensión de evitación tuvo una relación positiva con la sintomatología depresiva, así como lo afirman una serie de investigaciones previas (Fraley et al., 2011; González \& Méndez, 2006; Kamkar et al., 2012; Pardo et al., 2004). Asimismo, hubo asociación entre evitación y ansiedad rasgo - ansiedad estado, de manera coherente 
con lo esperado teóricamente (Cooper et al., 1998; Lee \& Hankin, 2009; Pacheco \& Ventura, 2009).

Sin embargo, respecto de la dimensión de ansiedad, no tuvo asociación ni con la sintomatología depresiva ni con la ansiedad estado y rasgo. Estos resultados pueden ser explicados desde diversos factores, aún cuando son propuestos de modo tentativo, considerando la poca disponibilidad de evidencia teórico-empírica en nuestro país sobre la medición del apego en la adolescencia a través de cuestionarios de autorreporte.

Se tienen antecedentes que la adaptación del ECR-RC realizada por Brenning et al., (2011) para población adolescente belga, sí obtuvo asociaciones en las líneas esperadas con la dimensión de ansiedad, por lo que es posible que factores de carácter sociocultural puedan estar moderando estos resultados. Un primer factor, guarda relación con la cultura europea, la cual posee valores intrínsecos relativos a una mayor autonomía en sus individuos, facilitando procesos de autoexpresión personal e identidad que podrían favorecer la identificación de aspectos internos en dichos adolescentes. Esto, versus la cultura latinoamericana, que mantiene valores tradicionales ligados a un mayor tiempo de permanencia de la población de adolescentes en sus hogares, y dependencia económica de los padres. Esto, podría explicar que los ítems destinados a evaluar ansiedad, más que ser interpretados como un reflejo de inseguridad en el apego, sean expresión de necesidades legitimadas culturalmente $y$ que por ende, no tendrían una asociación con síntomas psicopatológicos, como ocurrió en este caso.

El segundo, concierne a la redacción de los ítems, la cual pudo haber generado confusión en los participantes. Mikulincer y Shaver (2007), refieren que la redacción inversa de los ítems hace que la subescala de ansiedad sea más susceptible al sesgo de respuesta. De acuerdo a la etapa evolutiva en que se encuentran los adolescentes, se espera en ellos el desarrollo del pensamiento formal, el cual, les otorgaría una mayor capacidad de reflexión permitiéndole razonar con una mayor complejidad e integración de variados elementos (Allen, 2008). Considerando el rango etario de nuestro estudio (12 a 17 años) se podría desprender que, los adolescentes de mayor edad, podrían encontrarse en una etapa de incipiente desarrollo del pensamiento abstracto, aún no consolidado dificultando el desarrollo de mayor capacidad de análisis y la comprensión de aquellos ítems que contengan una redacción compleja. En ese sentido, quienes se sitúan en rangos de menor edad, podrían encontrarse incluso en una etapa de desarrollo cognitivo anterior, o de transición hacia el pensamiento formal, predominando las funciones del pensamiento concreto, con la capacidad para integrar información no desarrollada del todo (Moretti \& Peled, 2004) haciendo más limitada la comprensión para los adolescentes en relación a dichos ítems.

El tercero, está relacionado con las debilidades propias de los cuestionarios de autorreporte, ya que quienes los contestan pueden estar influenciados en gran medida por la deseabilidad social. De acuerdo a lo anterior, se requiere entonces, de un nivel de autoconocimiento e introspección para responder el cuestionario, especialmente, en los ítems que miden ansiedad, ya que esta dimensión estaría relacionada con emociones y vivencias internas, a diferencia de los reactivos de evitación que parecieran estar más vinculados a conductas concretas, que se esperaría fueran de más fácil detección para los jóvenes. Si bien es cierto, la literatura internacional (BartleHaring, 1997; Marcia, 1983; Martínez, 2007) indica que el pensamiento en el adolescente le permite llegar a ciertas conclusiones luego de una introspección personal, éstas estarian teñidas por 
procesos propios e inherentes a su etapa evolutiva, tales como: búsqueda de la identidad, el logro de la autonomía, el egocentrismo y el logro de la independencia de figuras significativas como los padres. En ese ámbito, Allen (2008) señala que el desarrollo cognitivo y emocional de los adolescentes les permite reflexionar y modificar sus estados de ánimos en relación al apego. De acuerdo a esto, esta característica pudiese generar mayor susceptibilidad o sesgos en sus respuestas (Soto, John, Gosling \& Potter, 2008), la que es una de las limitaciones del presente estudio en la medición del apego mediante el autorreporte.

Considerando estos antecedentes, se propone para futuras investigaciones la revisión de la subescala de ansiedad del ECR-RS, dado que si bien los ítems se agrupan como un solo factor y poseen una alta consistencia interna, no queda claro que efectivamente corresponda a la dimensión de ansiedad ante el abandono Por ello, se sugiere reformular la redacción de tales ítems para favorecer su comprensión y pertinencia a la realidad chilena. Asimismo, aumentar el número de ítems orientados a medir esta dimensión con la finalidad de pesquisar un mayor rango de signos que den cuenta de ansiedad en el apego en la población adolescente. En cuanto a la subescala de evitación, se recomienda la reformulación de los ítems 5 y 6 para que discriminen y carguen efectivamente en una y no en ambas dimensiones. Finalmente, en términos de mejorar las condiciones de aplicación para detectar de mejor forma lo que se está midiendo, se sugiere efectuar la aplicación del ECR-RS en contextos reducidos que propicien, efectivamente, la introspección en los adolescentes.

Pese a las limitaciones del presente estudio, este sienta las bases para contar con un instrumento para medir el apego adolescente en Chile desde una perspectiva dimensional. Se demuestra que, es posible abordar la dimensión de evitación reflejado en las buenas propiedades psicométricas encontradas, consistencia interna y correlaciones significativas con constructos teóricos relacionados. Respecto a la dimensión de ansiedad, nuestro estudio representa un avance en la detección de las limitaciones más significativas en el ECRRS, facilitando un punto de partida a futuros investigadores que deseen aportar en esta línea investigativa, implementando mejoras en función de estos antecedentes, en la medición del apego en este grupo etario.

En síntesis, si bien los resultados obtenidos no concuerdan del todo con lo hipotetizado, representan igualmente un avance en el conocimiento, al abrir una línea de investigación poco explorada en nuestro país, como lo es la evaluación del apego en una etapa importante de la vida del ser humano como es la adolescencia. 


\section{REFERENCIAS}

Ainsworth, M., Blehar, M., Waters, E., \& Wall, S. (1978). Patterns of attachment: A psychological study of the strange situation. New Jersey: Erlbaum.

Ainsworth, M., \& Bowlby, J. (1991). An ethological approach to personality development. American Psychologist, 46(4), 333-341. doi: 10.1037/0003066X.46.4.333

Allen, J. (2008). The attachment system in adolescence. J. Cassidy \& P. R. Shaver (Eds.), Handbook of attachment. Theory, research and clinical applications .Nueva York: The Guildford Press.

Bartholomew, K., \& Horowitz, L. (1991). Attachment styles among young adults: a test of a four-category model. Journal of Personality and Social Psychology, 61, 226-244. doi: 10.1037/0022-3514.61.2.226

Bartle - Haring, S. (1997). The relationships among parent- adolescent differentiation, sex role orientiation and identity development in late adolescence and early adulthood. Journal of Adolescence, 20, 553-565. doi: 10.1006/jado.1997.0109

Bentler. P. M. (2005). EQS 6 structural equations program manual. Encino, CA: Multivariate Software (www.mvsoft.com).

Blatt, S. J. \& Levy, K. N. (2003). Attachment Theory, Psychoanalysis, personality development, and psychopathology. Psychoanalytic Inquiry. 23, 102-150. doi: 10.1080/07351692309349028

Bowlby, J. (1980). La Pérdida Afectiva. Buenos Aires: Paidós.

Bowlby, J. (1986). Vínculos Afectivos: Formación, desarrollo y pérdida. Madrid: Morata.

Brando, M., Valera, J., Y Zarate, Y. (2008). Estilos de apego y agresividad en adolescentes. Segunda Época, 27(1), 1642.

Brennan, K., Clark, C., \& Shaver, P. (1998). Self-report measurement of adult attachment: An integrative overview. In J. A. Simpson \& W. S. Rholes (Eds.), Attachment Theory and close relationships (pp.46-76). New York: Guilford Press.

Brenning, K., Soenens, B., Braet, C., \& Bosmans, G. (2011). An adaptation of the Experiences in Close Relationships ScaleRevised for use with children and adolescents. Journal of Social and
Personal Relationship, 28(8), 1048-1072. doi: $10.1177 / 026540751140241$

Cassidy, J. (2008). The nature of the child's ties. In J. Cassidy \& P. R. Shaver (Eds.), Handbook of Attachment Theory, research and clinical applications. Nueva York: The Guildford Press.

Cooper, M. L., Shaver, P. R., \& Collins, N. L. (1998). Attachment styles, emotion regulation, and adjustment in adolescence. Journal of Personality and Social Psychology, 74(5), 1380- 1397.

DeKlyen, M., \& Greenberg, M. (2008). Attachment and psychopathology in childhood. In J. Cassidy y P. R. Shaver (Eds.), Handbook of Attachment. Theory, research and clinical applications (pp. 637665). New York: The Guildford Press.

Delgado, I., Oliva, A., \& Sánchez-Queija, I. (2011). Apego a los iguales durante la adolescencia y la adultez emergente. Anales de Psicología, 27, 155-163. doi: 10.6018/27.1.113581

Donbaeka, F. D., \& Elklitb, A. (2013). A validation of the Experiences in Close Relationships-Relationship Structures scale (ECR-RS) in adolescents. Attachment \& Human Development. doi: 10.1080/14616734.2013.850103

Escobar, M., \& Santelices, P. (2013). Attachment in adopted adolescents. National adoption in Chile. Childrens and Youth Service Review, 35, 488-492.

Fraley, C., Waller, G., \& Brennan, A. (2000). An item-response theory analysis of self-report measures of adult attachment. Journal of Personality and Social Psychology, 78, 350-365.

Fraley, C., Heffernan, M., Vicary, A., \& Brumbaugh, C. (2011). The experience in close relationships-relationship structures questionnaire: A method for assesing attachment orientations across relationships. Psychological Assessment, 23(3), 615-125. doi: 10.1037/a0022898

Galán, A. (2010). El apego. Más allá de un concepto inspirador. Revista de la Asociación Española de Neuropsiquiatría, 30(108), 581-595.

Garrido-Rojas, L. (2006). Apego, emoción y regulación emocional. Implicaciones para la salud. Revista Latinoamericana de Psicología, 38(3), 493-507.

George, C., Kaplan, N., \& Main, M. (1985). Adult Attachment Interview. Unpublished 
manuscript, University of Berkeley, California

Gempp, R., Avendaño, C. Y Muñoz, K. (2004) Normas y punto de corte para la Escala de Depresión del centro para estudios epidemiológicos (CES-D) en población juvenil chilena. Terapia Psicológica, 22(2), 145-156.

González, L. Y Méndez, L. (2006). Relación entre autoestima, depresión y apego en adolescentes urbanos de la comuna de Concepción, Chile. Terapia Psicológica, 24(1), 5-14.

Harel, O., Zimmerman, R., \& Dekhtyar, O. (2008). Approaches to the handling of missing data in communication research. En A. F. Hayes, M. D. Slater \& L. B. Snyder (Ed.).. Advanced data analysis methods for communication research (pp. 349-371). Thousand Oaks: Sage Publications.

Hazan, C. \& Shaver, P. (1987). Romantic love conceptualized as an attachment process. Journal of Personality and Social Psychology, 52, 511-524.

Kamkar, K., Doyle, A., \& Markiewicz, D. (2012). Insecure Attachment to parents and depressive symptoms in early adolescence: Mediating roles of atributtions and self-esteem. International Journal of Psychological Studies, 4(2) 3-18. doi:10.5539/ijps.v4n2p3

Kline, R. B. (2005). Principles and practice of structural equation modeling (2nd Ed.). New York: Guilford Press.

Kobak, R., Sudler, N., \& Gamble, W. (1991). Attachment and depressive symptoms during adolescence: a developmental pathways analysis. Development Psychopathology, 3, 461-474. doi: 10.1017/S095457940000763X

Lecannelier, F. (2002). La entrevista de apego en niños/ Child attachment interview - CAl. Terapia Psicológica, 20(1), 53-60.

Lee, A., \& Hankin, B. (2009). Insecure attachment, dysfunctional attitudes, and low self-steem predicting prospective symptoms of depression and anxiety during adolescence. Journal of Clinical Child \& Adolescencent Psychology, 38(2), 219-23. Doi: 10.1080/15374410802698396

Loinaz, I., Echeburúa, E. \& Ullate, M. (2012). Estilo de apego, empatía y autoestima en agresores de pareja. Terapia psicológica, $30(2), 61-70$.
Marcia, J. (1983). Some Directions for the investigation of ego development in Early Adolescence. The Journal of Early Adolescence, 3(3), 215-223.

Martínez, L. (2007). Mirando al Futuro: Desafíos y oportunidades para el desarrollo de los adolescentes en Chile. Pshyke. 16(1). 3$14 . \quad$ doi: $0.4067 / S 0718$ 22282007000100001

Martínez, C., \& Santelices, M. (2005) Evaluación del apego en el adulto: Una revisión. Psykhe, 14(1), 181-191. doi: 10.4067/S0718-22282005000100014

McElhaney, K. B., Allen, J. P., Stephenson, J. C., \& Hare, A. L. (2009). Attachment and autonomy during adolescence. En Lerner, R. \& Steinberg, L. (Eds.), Handbook of adolescent psychology (pp. 358-403). New Jersey: John Wiley \& Sons.

Mikulincer, M., \& Shaver, P. R. (2007). Attachment in adulthood: Structure, dynamics, and change. New York: Guilford.

Moretti, M., \& Peled, M. (2004). Adolescentparent attachment: Bonds that support healthy development. Paedriatics \& Child Health, 9(8), 551-555.

Oliva, A. (2004). Estado actual de la Teoría del Apego. Revista de Psiquiatría y Psicología del Niño y del Adolescente, 4(1), 65-81.

Oliva, A. (2011). Apego en la adolescencia. Acción Psicológica, 8(2), 56-65.

Pardo, G., Sandoval, A., Y Umbarila, D. (2004). Adolescencia y depresión. Revista Colombiana de Psicología, 13, 13-28.

Pacheco, B., Y Ventura, T. (2009). Trastorno de ansiedad por separación. Revista Chilena de Pediatría, 28(2), 109-119.

Rojas-Barahona, C., Zegers, B., \& Förster, C. (2009) La Escala de Autoestima de Rosenberg: Validación para Chile en una muestra de jóvenes adultos, adultos y adultos mayores. Revista Médica de Chile, $137,791-800$.

Román, M. (2011). Metodologías para la evaluación del apego infantil: De la observación de conductas a la exploración de las representaciones mentales. Acción Psicológica, 8(2), 27-38.

Schore, A. (2009). Advances in neuropsychoanalysis, Attachment Theory, and trauma research: Implications for self psychology. Psychoanalytic Inquiry, 22, 433-484.

Shmueli-Goetz, Y. (2001). Child Attachment Interview: Development and validation. 
Unpublished doctoral dissertation. University College London, England.

Smith- Castro, V. \& Molina, M. (2011). La entrevista cognitiva: guía para su aplicación en la evaluación y mejoramiento de instrumentos de papel y lápiz. (Cuaderno Metodológico 5). San José, Costa Rica: Universidad de Costa Rica, Instituto de Investigaciones Psicológicas.

Soto, C., John, P., Gosling, S., \& Potter, J. (2008). The developmental psychometrics of Big Five Self-Reports: acquiescence, factor structure, coherence, and differentiation from ages 10 to 20. Journal of Personality and Social Psychology, 94(4), 718-737. doi: 10.1037/00223514.94.4.718.

Spencer, R., Guzmán, M., Fresno, A., Y Ramos, N. (2012). Validación chilena del Cuestionario de Evaluación del Apego Romántico Experiences in Close Relationships (ECR): análisis de la validez de criterio. Terapia psicológica, 31(3), 313324.

Steele, H., \& Steele, M. (2005). The construct of coherence as an indicator of attachment security in middle childhood: The Friends and Family Interview. En K. A. Kerns \& R. A. Richardson (Eds.), Attachment in middle childhood. Nueva York: Guilford Press.

Tabachnick, B., \& Fidell, L. (2007). Using multivariate statistics. Boston: Pearson/Allyn \& Bacon.

Target, M., Fonagy, P., \& Shmueli-Goetz, Y. (2003).Attachment representations in school-age children: The development of the Child Attachment Interview (CAl). Journal of Child Psychotherapy, 29(2), 171-186.

Van ljzendoorn, M., \& Bakermans-Kranenburg, M., (2003). Attachment disorder and disorganized attachment: Similar and different. Attachment and Human Development, 5(3), 313-320.

Vera-Villarroel, P., Celis-Atenas, K., CórdovaRubio, N., Buela-Casal, G., \& Spielberger, Ch.D. (2007). Preliminary analysis and normative data of the State-Trait Anxiety Inventory (STAI) in adolescent and adults of Santiago, Chile. Terapia Psicológica, 25,155-162.

West, M., Rose, M., Spreng, S., Sheldon-Keller, A., \& Adam, K. (1998). Adolescent Attachment Questionnaire: a brief assessment of attachment in adolescence.
Journal of Youth and Adolescence, 27, 661-673.

Wilkinson, R. (2004). The role of parental and peer attachment in the psychological health and self-esteem of adolescents. Journal of Youth and Adolescence, 33(6), 479-493.

Zegers B, Rojas-Barahona C., Föster C. (2009). Validez y confiabilidad del índice de Satisfacción Vital (LSI-A) de Neugarten, Havighrust \& Tobin en una muestra de adultos $y$ adultos mayores en Chile. Terapia Psicológica, 27(1), 15-26. 